\title{
Supercontinuum Generation in Highly Nonlinear PQF with Two Zero Dispersion Wavelength
}

\author{
Sivacoumar Rajalingam*, Zachariah C. Alex \\ School of Electronics Engineering, Vellore Institute of Technology (VIT), India
}

Received September 9, 2019; Revised December 19, 2019; Accepted December 24, 2019

Copyright $@ 2019$ by authors, all rights reserved. Authors agree that this article remains permanently open access under the terms of the Creative Commons Attribution License 4.0 International License

\begin{abstract}
An optical input pulse with a duration of nanosecond to femtosecond propagates through a dispersive nonlinear medium, it is subject to an extreme spectral broadening termed as white light supercontinuum (SC). In PCF, dispersion characteristics can be modified by the waveguide geometric parameters and thereby the nonlinear dynamics can be altered. In this article, the fiber parameters like core diameter, doping percentage, and pitch are varied and analyzed to achieve a conclusive zero-dispersion wavelength (ZDW) and a high nonlinearity. The numerical study presents different designs of solid-core photonic quasi-crystal fiber (PQF) and selection of highly nonlinear fiber with an effective area of $1.14 \mu \mathrm{m}^{2}$ and nonlinearity of $196 \mathrm{~W}^{-1}-\mathrm{km}^{-1}$ at $780 \mathrm{~nm}$ exhibiting two-zero dispersion wavelength for supercontinuum generation in different regimes. The results exhibit a significant pulse broadening effect when the pump pulse (FWHM) was varied from 25 fs to 100 fs. Similarly, the influences of the pulse energy and peak average power on the bandwidth of the generated SC are studied
\end{abstract}

Keywords Supercontinuum Generation, Photonic Quasi-crystal Fibers, Zero-dispersion Wavelength, Nonlinearity, Soliton Fission

\section{Introduction}

Supercontinuum generation was first recognized in 1969, when Alfano et al., was engaged in characterizing crystals and glasses. Alfano et al., studied the effect of SPM by exciting the nonlinear materials using picosecond pulse from the frequency doubled (5300 §) Nd: glass laser[1]. However, in early 1968, the role of SPM in glasses and the underlying effects in spectral broadening and linear frequency chirp have been demonstrated. Supercontinuum generation is generally a blend of many nonlinear effects, which include stimulated Raman scattering (SRS), stimulated Brillouin scattering (SBS), the optical Kerr effect, four-wave mixing (FWM), and self-phase modulation (SPM), occurring simultaneously[2-4].

In 1973 Hasegawa et al., proposed a key concept in SC generation related to the balance between the SPM and anomalous dispersion with respect to the pump power and the pulse width. However, a pulse with any reasonable shape will evolve into solitons [5]. Launching the optical pulse of any arbitrary shape and amplitude near the minimum dispersion region ie. zero dispersion wavelength (ZDW) would generate solitons. To exhibit a smooth spectral profile of the generated supercontinua, Washio et al., demonstrated the significance of pumping in the anomalous dispersion region using Q-switched and mode-locked Nd:YAG solid state laser at $1.34 \mu \mathrm{m}$ wavelength [6]. By increasing the fiber length, the required pump power can be minimized with efficient FWM in the region of $\mathrm{ZDW}$.

Photonic band-gap fibers (PBGFs) display fascinating properties, making them attractive especially for nonlinear applications. Since PBGF exhibits one ZDW in each transmission window, it has the potential to generate a broad SC. Bétourné et al., have demonstrated the SC generation using nanosecond pump pulse with a flat spectrum. The generated spectrum mainly ranged from $1050 \mathrm{~nm}$ to $1580 \mathrm{~nm}$ with the cancellation of soliton self-frequency shift (SSFS) at the band edges[7]. Zhang et al., has numerically investigated the liquid filled hollow core-photonic crystal fiber (HC-PCF) for SC generation. Normally, HC-PCF is not widely used for SC generation. Rather, HC-PCF core is filled with highly nonlinear liquid or gases for nonlinear applications. The investigation indicates the fact, when the central core is infiltrated with nitrobenzene and carbon disulfide, the nonlinearity factor $(\gamma)$ increases drastically to a value of $2.4 / \mathrm{W} / \mathrm{m}$. As $\gamma$ increases, the SC generation drastically broadens from 700 $\mathrm{nm}$ to $2500 \mathrm{~nm}$ [8]. The procedure to fill the hollow-core PCF with liquids is a tedious task. Gases offer numerous advantages over their counterpart- the solid state materials, as they degrade due to the optical damage at high intensities. The advantage of using gases is its nonlinearity 
and dispersion can be tuned with varying pressure and gas mixture. Russell et al., has demonstrated a widely tunable hydrogen-filled Kagome structure PCF, generating spectrum extending from vacuum ultraviolet to the near infrared region[9]. Tong Tong et al., have proposed a photonic quasicrystal fiber with background material as chalcogenide glass $\left(\mathrm{As}_{2} \mathrm{Se}_{3}\right)$. The proposed $\mathrm{PQF}$ has large air holes, which influences the modal confinement and is also optimized to exhibit dual ZDW. Hence, the effective mode area of the design is very small leading to high nonlinearity with promising application in SC generation [10]

\section{Design Aspects of Photonic Quasi-Crystal (PQF) Fiber}

After the advent of PCFs by Russell et al, the impact of various structural parameters is taken into account and its influences are investigated in SC generation in spectral and temporal coherence[11]. One common challenge the designer facesis the pump wavelength, which should be matched nearer to the ZDW. In most situations, novel fiber designs ZDWs are optimized to match with the available pump sources.Using a small hole diameter to pitch ratio and minimizing the core diameter, the dispersion profile can be befitted to have two-ZDWs. The small core along with two-ZDW can improve intensity-dependent nonlinear processes such as FWM and higher-order dispersion effects[12].

We have illustrated the transverse cross section of five-fold solid core PQF in Figure 1. Quasicrystals are crystals with long-range translational and orientational orders [13-14]. However, the translational order is not periodic and the structure does not necessarily have crystallographic rotational point symmetry. These structures reveal more significant attributes than the stereotyped PCFs, due to the degree of freedom that has been concealed in aperiodic structures. The proposed PQF structure is formed by two types of rhombic tiles: thin tile with angles of $(\pi / 5)$ and $(4 \pi / 5)$, and thick tile with angles of $(2 \pi / 5)$ and $(3 \pi / 5)$. The resulting connected space-filling packing of unit cells is called Penrose lattice [15].

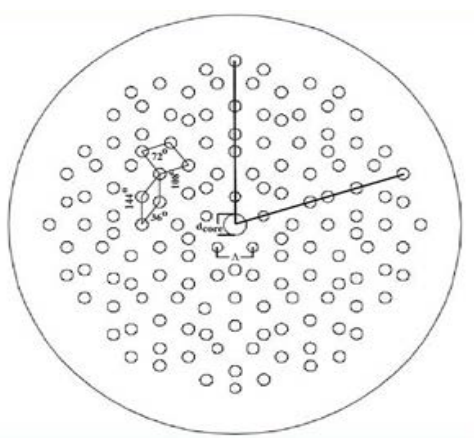

Figure 1. The geometrical structure of the proposed five-fold symmetric PQF

In the study, initially the inner core of PQF is maintained with a diameter of $1.0 \mu \mathrm{m}\left(\mathrm{d}_{\text {core }}\right)$ and is doped with very low concentration of $\mathrm{GeO}_{2}$ contributing to an index difference of $1-2 \%$. In the lattice design, the first ring of air holes is formed by maintaining a pitch of $2.0 \mu \mathrm{m}(\Lambda)$. The adjacent rings in the cladding are formed by quasi-lattice distribution with a 5-fold symmetry. The air hole in the cladding depresses the average index of the cladding region and confines light within the central core [16]. The analysis of space-filling modes in the fiber is generally complicated, but by applying the approximation the relative index difference is very less, the fibers satisfy the weakly guiding modes. The dispersion parameter

$$
D(\lambda)=-(\lambda / c)\left(d^{2} n_{\text {eff }} / d \lambda^{2}\right)
$$

Eq. (1) is derived using the knowledge of the effective refractive index versus the wavelength obtained by the FEM approach. On the basis of the Penrose quasi-lattice distribution, the dispersion curves are tailored by changing the geometric parameters. 


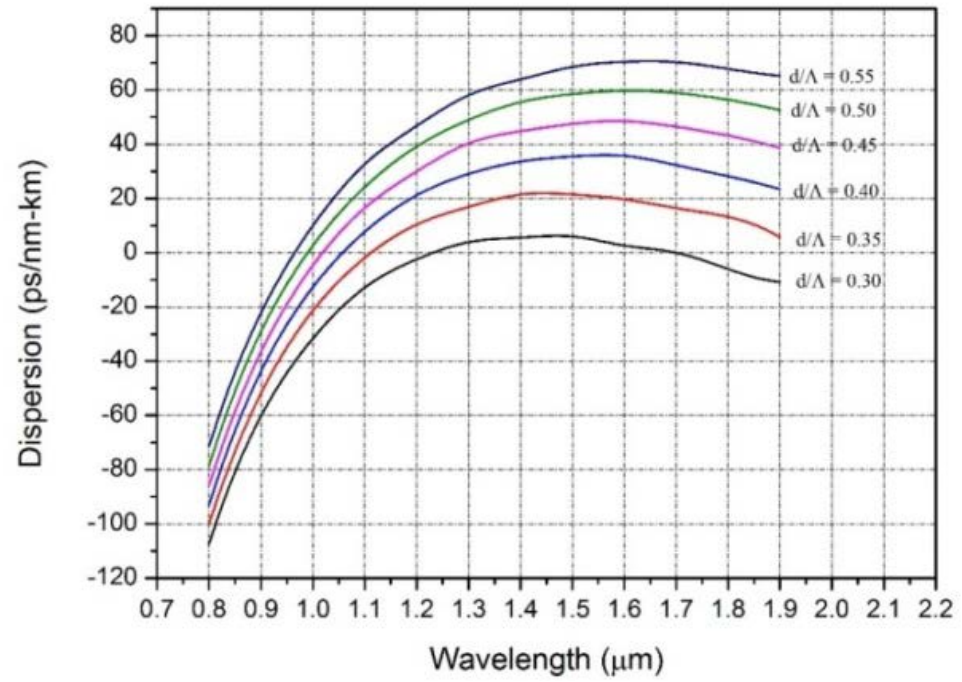

Figure 2a. Dispersion profile of the PQF, with core diameter $1 \mu \mathrm{m}$, core doping with a fractional index difference of $2 \%$ and the pitch is maintained at $2 \mu \mathrm{m}$. The diameters of the air holes are varied from $0.5 \mu \mathrm{m}$ to $1.1 \mu \mathrm{m}$

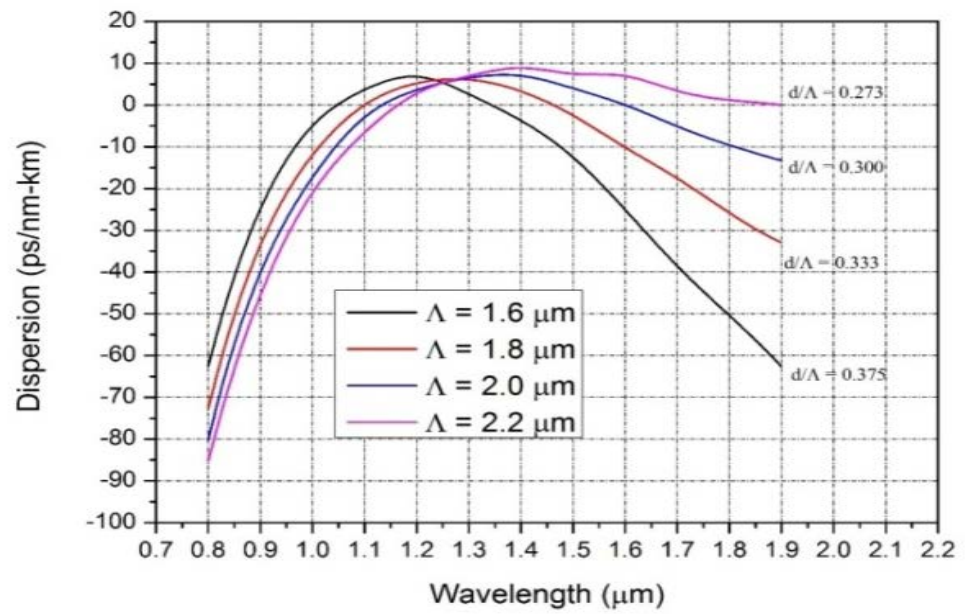

Figure 2b. Dispersion profile of the PQF, with core diameter $1 \mu \mathrm{m}$, the core is undoped and the diameters of the air holes are maintained at $0.6 \mu \mathrm{m}$. The pitch is varied from $1.6 \mu \mathrm{m}$ to $2.2 \mu \mathrm{m}$

The family of dispersion curve (Figure 2a) reveals the fact when the $d / \Lambda$ ratio increases the dispersion curve and the curve become flattering after $1.3 \mu \mathrm{m}$ with single ZDW. The ZDW also shifts toward visible region as the $\mathrm{d} / \Lambda$ ratio increases. On the contrary, as the $d / \Lambda$ approaches 0.3 , the dispersion curve yields two ZDWs $(1.231 \mu \mathrm{m}$ and $1.703 \mu \mathrm{m})$. In 2004, Hilligsøe et al., has demonstrated numerically and experimentally that SC generation in two closely spaced ZDWs in PCFs has smooth and stable spectral parts [17].To obtain a greater smoothness and stability in output spectra, the structural parameter pitch $(\Lambda)$ is considered for optimizing the two ZDW ranges. The undoped core diameter is kept constant at $1 \mu \mathrm{m}$ and with cladding air hole diameter at $0.6 \mu \mathrm{m}$. The pitch was varied from $1.6 \mu \mathrm{m}$ through $2.2 \mu \mathrm{m}$. From the study, it was found that the separation between the ZDW increased as the $d / \Lambda$ decreased from 0.375 to 0.273 . In the literature cascading two ZDW PCFs placed wide apart have demonstrated very flat spectra. In 2016, Zhang et al., cascaded a dual ZDW PCF to single ZDW PCF, and pumped using a picosecond laser to demonstrate an ultraflat SC generation with $3 \mathrm{~dB}$ bandwidth of $560 \mathrm{~nm}(1070 \mathrm{~nm}-1630 \mathrm{~nm})$ (Zhang et al., 2016)

In Figure 2b, the ZDW1 falls at $1.044 \mathrm{~mm}, 1.097 \mathrm{~mm}$, $1.132, \mathrm{~mm}$ and $1.162 \mathrm{~mm}$ and the ZDW2 shifts apart as the $\mathrm{d} / \Lambda$ increases (1.336, 1.451, 1.599 and $1.883 \mathrm{~mm})$. The pump pulse wavelength should be in the close vicinity of the ZDW as it plays a vital role in the dynamics of the SC generation. Here, the ZDWs are optimized to be closely matched to the wavelength of Q-switched Nd:YAG lasers. In optimizing the zero dispersion in fibers, doping of core can achieve a considerable lateral shift. Here, the PQF geometrical parameters are kept constant, with the core diameter maintained at $1 \mathrm{~mm}$ and the diameter of air holes at $0.6 \mathrm{~mm}$. The core doping percentage is varied such that the fractional refractive index between the cores is maintained from 0 to $1 \%$. The plot (Figure 3a) clearly illustrates that the ZDWs are shifted laterally maintaining the peak dispersion value almost constant. 


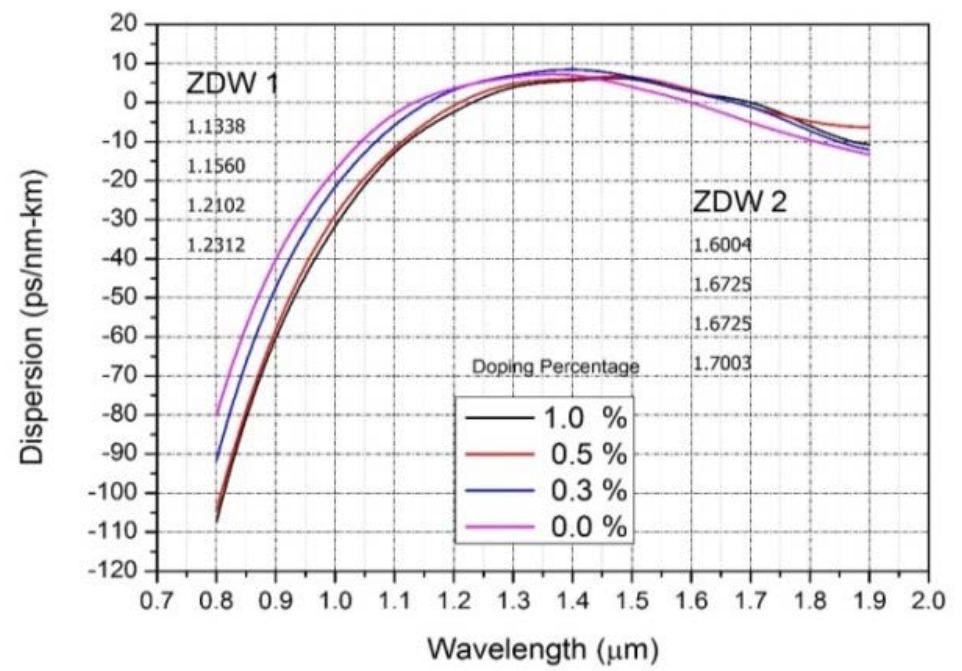

Figure 3a. Dispersion profile of the PQF, with core diameter at $1 \mu \mathrm{m}$, the diameters of the air holes maintained at $0.6 \mu \mathrm{m}$. The core doping percentage is varied from 0 to $1 \%$

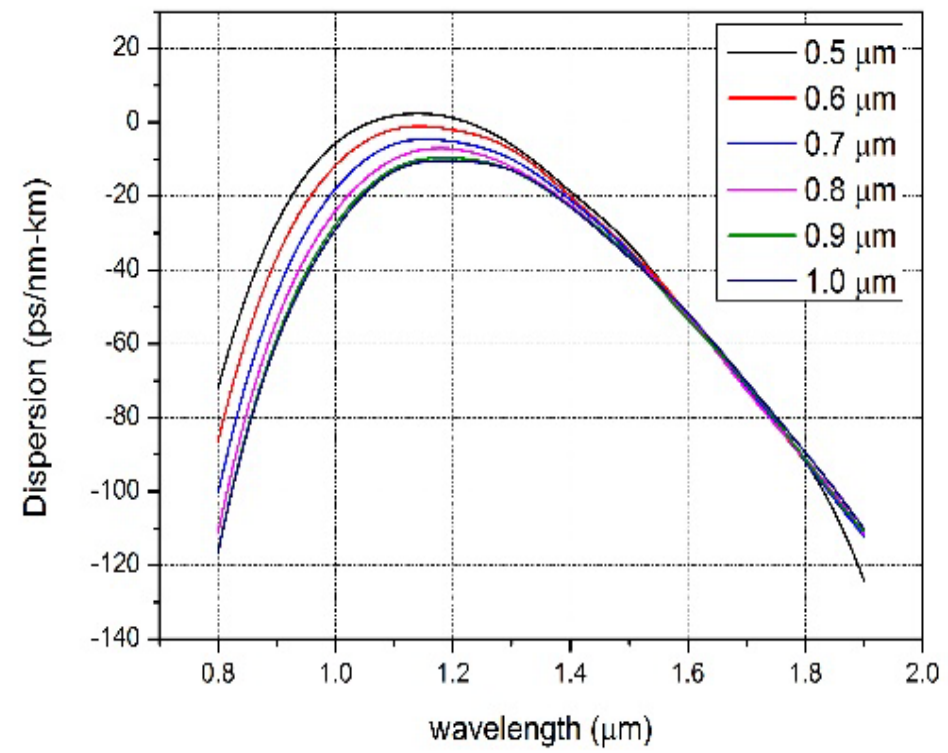

Figure 3b. The core is undoped and the diameters of the air holes are maintained at $0.6 \mu \mathrm{m}$ and pitch is at a constant value of $1.4 \mu \mathrm{m}$. The core diameter is varied from $0.5 \mu \mathrm{m}$ to $1 \mu \mathrm{m}$.

The nonlinearity coefficient is inversely proportional to the effective mode area. A small effective mode area contributes toward the high power density of the input optical pulse. Nonlinear effects come to picture when the power density increases above a threshold limit [23]. In Figure $3 \mathrm{~b}$, dispersion curve shifts from anomalous region toward normal dispersion curve. When the core diameter decreases from $1.0 \mu \mathrm{m}$ to $0.5 \mu \mathrm{m}$, the effective mode area decreases rapidly. In Figure 4, for PQF with core diameter $0.5 \mu \mathrm{m}$ depicts the relation between the mode area and the optical nonlinearity. As the operating wavelength moved toward the visible region, the mode confinement became tight with very less $A_{\text {eff }}$ generating very high nonlinearity $\left(90.33 \mathrm{~W}^{-1} \mathrm{~km}^{-1}\right.$ at $\left.700 \mathrm{~nm}\right)$.

On the basis of the analysis, we choose three fibers for the SC generation with the parameters mentioned in Table 1 . The fiber parameters are chosen in such a way that the ZDW falls in the different regimes. The dispersion curve of the optimized PQF is plotted in Figure 5. The HNLF-1 dispersion curve ZDW1 is positioned at $836.7 \mathrm{~nm}$ and the second ZDW at $1645.2 \mathrm{~nm}$. The fiber HNLF-2 has positioned the ZDWs at $687.04 \mathrm{~nm}$ and $1589.2 \mathrm{~nm}$. The fiber HNLF-3 dispersion crosses the zero dispersion at $1049.4 \mathrm{~nm}$ and $1221.0 \mathrm{~nm}$. 
Before considering the general case, it is informational to concentrate on the impacts of second-order dispersion $\left(\beta_{2}\right)$ alone. The dispersion parameter is related to second-order dispersion (GVD) described by

$$
D(\lambda)=\frac{d \beta_{1}}{d \lambda}=-\frac{2 \pi c}{\lambda^{2}} \beta_{2}
$$

Nonlinear effects in fibers show clear impact depending on the sign of the second-order dispersion. When operated at a wavelength less than the ZDW of the fiber, the fibers display normal dispersion $\left(\beta_{2}>0\right)$. On the contrary, when operated at a wavelength greater than the ZDW, the fibers exhibit anomalous dispersion $\left(\beta_{2}>0\right)$. The anomalous region is of much significance as the fibers evoke nonlinear effects counterbalancing linear dispersive effect. The higher-order dispersion coefficient of the propagating wave can be deduced as follows:

$$
\begin{gathered}
\beta_{2}=\frac{d}{d \omega}\left[\frac{d k}{d \omega}\right]=-\frac{\lambda^{3}}{2 \pi c^{2}} \frac{d^{2} n_{e f f}}{d \lambda^{2}}, \\
\beta_{3}=\frac{d}{d \omega}\left[\frac{d^{2} k}{d \omega^{2}}\right], \beta_{m}=\frac{d}{d \omega}\left[\frac{d^{m-1} k}{d \omega^{m-1}}\right]
\end{gathered}
$$

where $\mathrm{k}$ is the wavenumber and $\omega$ is the angular frequency. In the following plot (Figure 7), the majority of the region is under anomalous dispersion region. It is quite obvious that PQF exhibits a flat negative GVD for a wider bandwidth. The bandwidth of negative dispersion for HNLF is approximately $902 \mathrm{~nm}, 808 \mathrm{~nm}$, and $171 \mathrm{~nm}$.

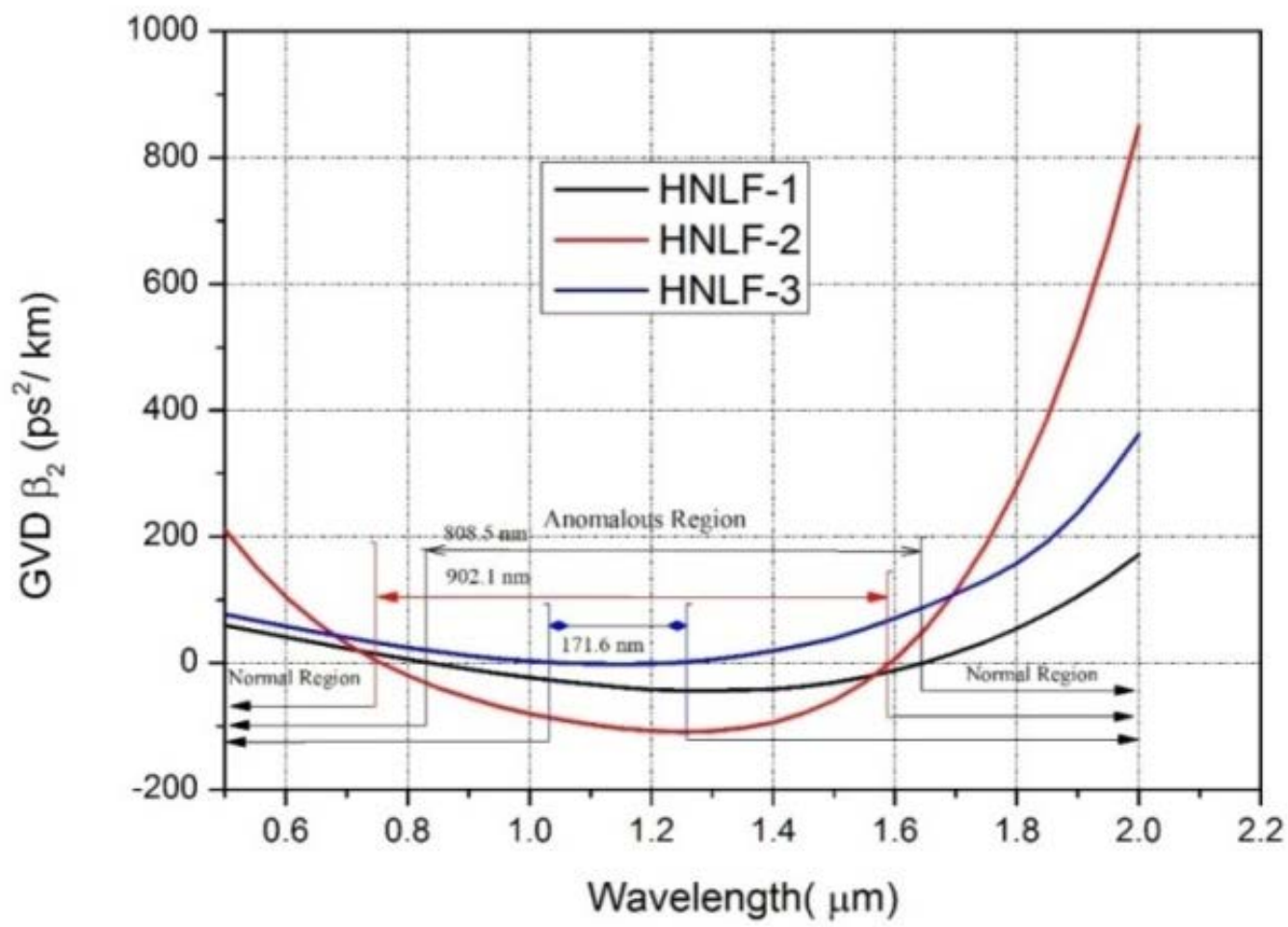

Figure 6. Calculated second-order dispersion curves GVD for the proposed PQF with the geometric parameters given in Table 1 


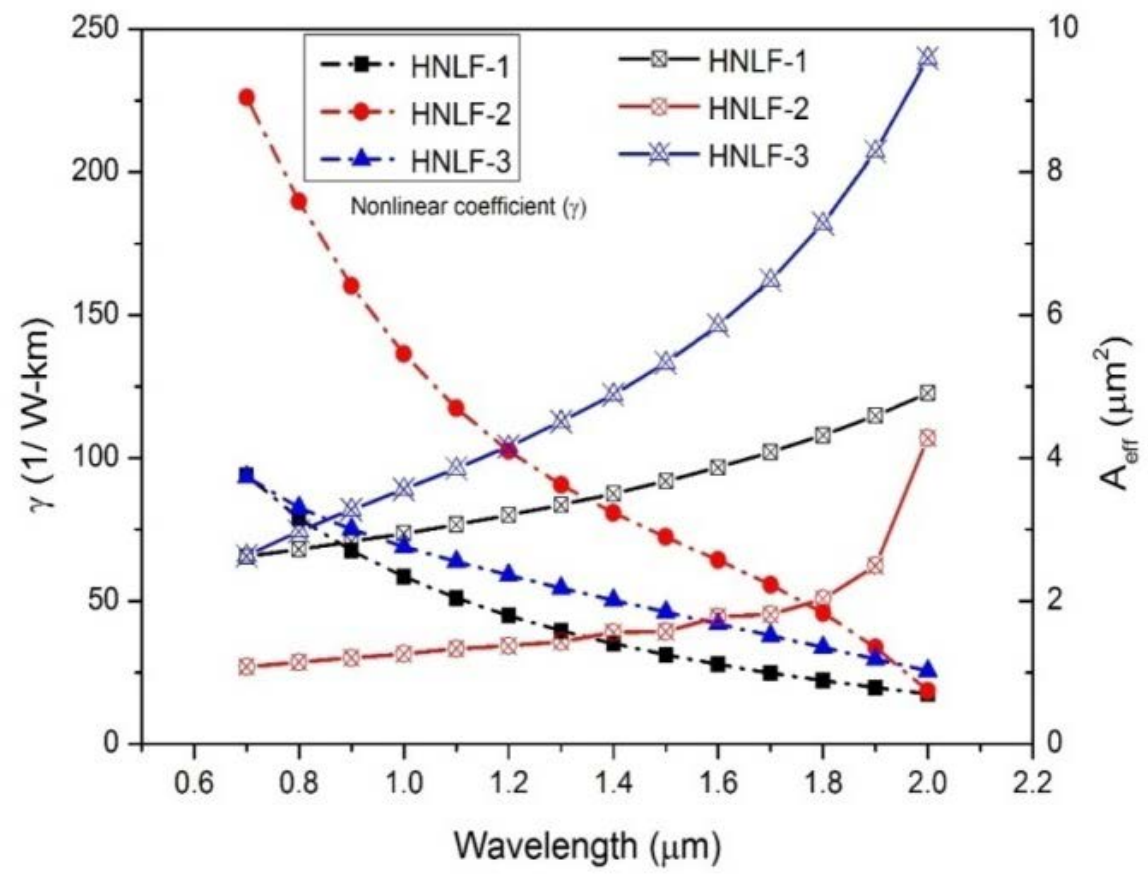

Figure 7. Variation of effective area of the proposed PQF with respect to wavelength and corresponding nonlinearity

$$
\gamma\left(\omega_{o}\right)=\frac{n_{2}\left(\omega_{o}\right) \omega_{o}}{c A_{\text {eff }}}
$$

It is obvious from the above equation (4), when the effective area of the fiber is less, the nonlinearity factor increases. The nonlinearity parameter for the HNLF is 75 $\left[\mathrm{W}^{-1} \mathrm{~km}^{-1}\right], 196\left[\mathrm{~W}^{-1} \mathrm{~km}^{-1}\right]$, and $69\left[\mathrm{~W}^{-1} \mathrm{~km}^{-1}\right]$ at the ZDW (Figure 8). In further analysis, these three fibers parameters are extensively studied and mostly HNLF-2 results are discussed since it has highest nonlinearity factor.

\section{Supercontinuum Generation in Different Regime}

By considering the width of the pump pulses, the generation of supercontinuum is classified under different propagation regimes. When the pump pulse width is in the order of femtosecond to picosecond, then the output spectra are greatly influenced by the solitons dynamics in boarding the spectra. If the pulse width increases $>100 \mathrm{ps}$ to nanosecond, then new frequency components are generated sustainably due to the modulation instability or FWM [18]. The dynamics of SCG in PCFs using femtosecond laser pulses firmly rely on the relative detuning between the ZDW of the fiber and the pump wavelength. From the previously reported results, it is observed that the SC spectra evolve differently based on the pump wavelength employed at anomalous or normal dispersion region of the fiber.

Now, we present the analysis of the numerical simulation using split-step Fourier method to solve the GNLSE. As given in Table 2, the fibers HNLF-1 and HNLF-2 are considered under the anomalous pumping regime. As the mode-locked Ti-sapphire laser with wide wavelength range (700 nm to $1300 \mathrm{~nm}$ ) is commercially available. Now-a-days these lasers comes with output pulse as short as few femto second to atto-second pulses. The pump wavelength $(780 \mathrm{~nm}$ and $840 \mathrm{~nm}$ ) for the numerical analysis is carefully selected to be near the ZDW wavelength of the proposed fiber. In the simulation, the injected optical pulse is a hyperbolic secant field with an average power of $42.6 \mathrm{~mW}$. The optical pulse has a repetition rate of $80 \mathrm{MHz}$, corresponding to FWHM of 50 fs with the pulse energy of $0.6 \mathrm{~nJ}$.

The spectral evolution is determined by the nonlinear factors in the proposed fiber; the simulation reproduces qualitatively the same characteristic spectra reported by the experimental studies in the literature[19]. It is quite complex to understand the fundamentals of physics involved in the generation of the SC. The evolution of the SC spectrum can be divided into three stages: initial higher-order soliton compression; soliton fission and dispersive wave generation; Raman self-frequency shift. Figure 8 clearly demonstrates the dynamics of SC at the initial phase where the spectral broadening is more symmetrical about the pump wavelength. The initial broadening appears at $0.4 \mathrm{~cm}$ and the strong temporal compression takes place within $1 \mathrm{~cm}$ of the fiber. 
Table 2. GVD and higher-order dispersion terms used in the analysis at the pump wavelength $840 \mathrm{~nm}$ and $780 \mathrm{~nm}$

\begin{tabular}{|c|c|c|}
\hline Dispersion Coefficient & HNLF-1 & HNLF-2 \\
\hline$\beta_{2}$ & $-5.2682 \times 10^{-4} \mathrm{ps}^{2} / \mathrm{km}$ & $-1.0622 \times 10^{-2} \mathrm{ps}^{2} / \mathrm{km}$ \\
\hline$\beta_{3}$ & $6.1432 \times 10^{-5} \mathrm{ps}^{3} / \mathrm{km}$ & $1.4577 \times 10^{-4} \mathrm{ps}^{3} / \mathrm{km}$ \\
\hline$\beta_{4}$ & $-7.4813 \times 10^{-9} \mathrm{ps}^{4} / \mathrm{km}$ & $5.2560 \times 10^{-8} \mathrm{ps}^{3} / \mathrm{km}$ \\
\hline$\beta_{5}$ & $5.9893 \times 10^{-11} \mathrm{ps}^{5} / \mathrm{km}$ & $-4.9900 \times 10^{-11} \mathrm{ps}^{5} / \mathrm{km}$ \\
\hline$\beta_{6}$ & $2.1295 \times 10^{-13} \mathrm{ps}^{6} / \mathrm{km}$ & $-5.5915 \times 10^{-14} \mathrm{ps}^{13} / \mathrm{km}$ \\
\hline$\beta_{7}$ & $-1.5572 \times 10^{-15} \mathrm{ps}^{7} / \mathrm{km}$ & $7.2346 \times 10^{-17} \mathrm{ps}^{7} / \mathrm{km}$ \\
\hline$\beta_{8}$ & $7.5803 \times 10^{-18} \mathrm{ps}^{8} / \mathrm{km}$ & $2.1865 \times 10^{-18} \mathrm{ps}^{8} / \mathrm{km}$ \\
\hline
\end{tabular}
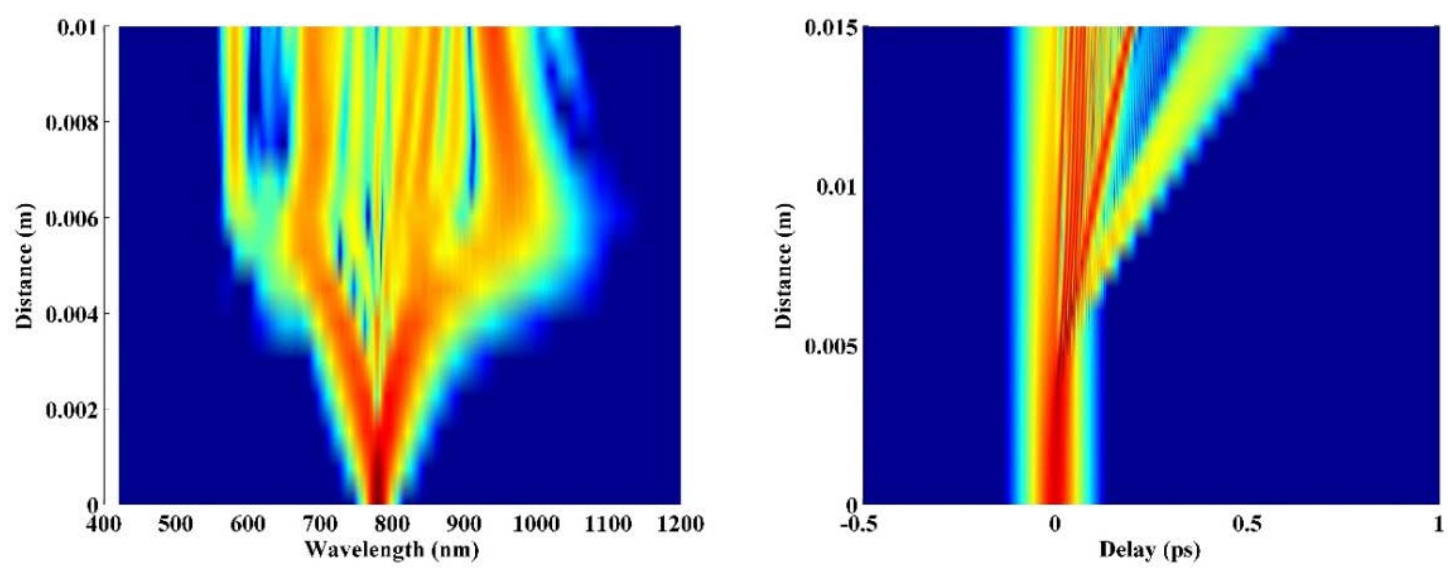

Figure 8. Initial stage symmetrical broadening with the first $0.4 \mathrm{~cm}$ for the proposed PQF HNLF-1

When the pulse progresses, the symmetrical broadening is further enhanced by the fission with distinct peaks arising toward higher and lower frequencies. These peaks further break down as low amplitude dispersive waves. As the propagation distance increases, the high-frequency peaks continue to expand while the low-frequency components cease to broaden. To understand the underlying soliton dynamics, the influences of individual nonlinear contributions are numerically examined. Higher-order solitons are sensitive to perturbations due to Raman scattering, higher-order dispersion, or modulation instability (MI). The formation of SC requires a high intense peak power for the input optical pulse, such that the order of the soliton is high enough to undergo fission. If the order of the soliton (N) is 1, the breaking of soliton may not happen (Figure 9). But higher-order solitons can decay into sub-pulses under the influence of Raman contribution and MI. This process is known as soliton fission. Usually at the point of maximum compression, the fundamental soliton will undergo fission giving rise to a fission length $\mathrm{L}_{\text {fiss }}$ given by $\mathrm{LD} / \mathrm{N}$. In the analysis for $\mathrm{N}=3, \mathrm{LD}=7.701 \mathrm{~cm}$, and a peak power of $596 \mathrm{~W}$, the fission length is calculated to be $2.56 \mathrm{~cm}$ (Figure 10b). A higher-order soliton of order $\mathrm{N}$ will emit $\mathrm{N}$ fundamental solitons, which are emitted in the decreasing order of peak intensity. These solitons then undergo SSFS, forming a continuum of solitons. Furthermore, they get shifted to different wavelengths due to the Raman contribution, and different temporal positions due to dispersion. If a soliton is propagating close to the ZDW, then some energy can be transferred into the normal dispersion regime known as a dispersive wave (DW). Normally DW generation requires phase-matching condition with the Raman-shifted solitons[18]. The overall result due to soliton fission, dispersive wave radiation, and SSFS will generate a broadband continuum, known as supercontinuum. 

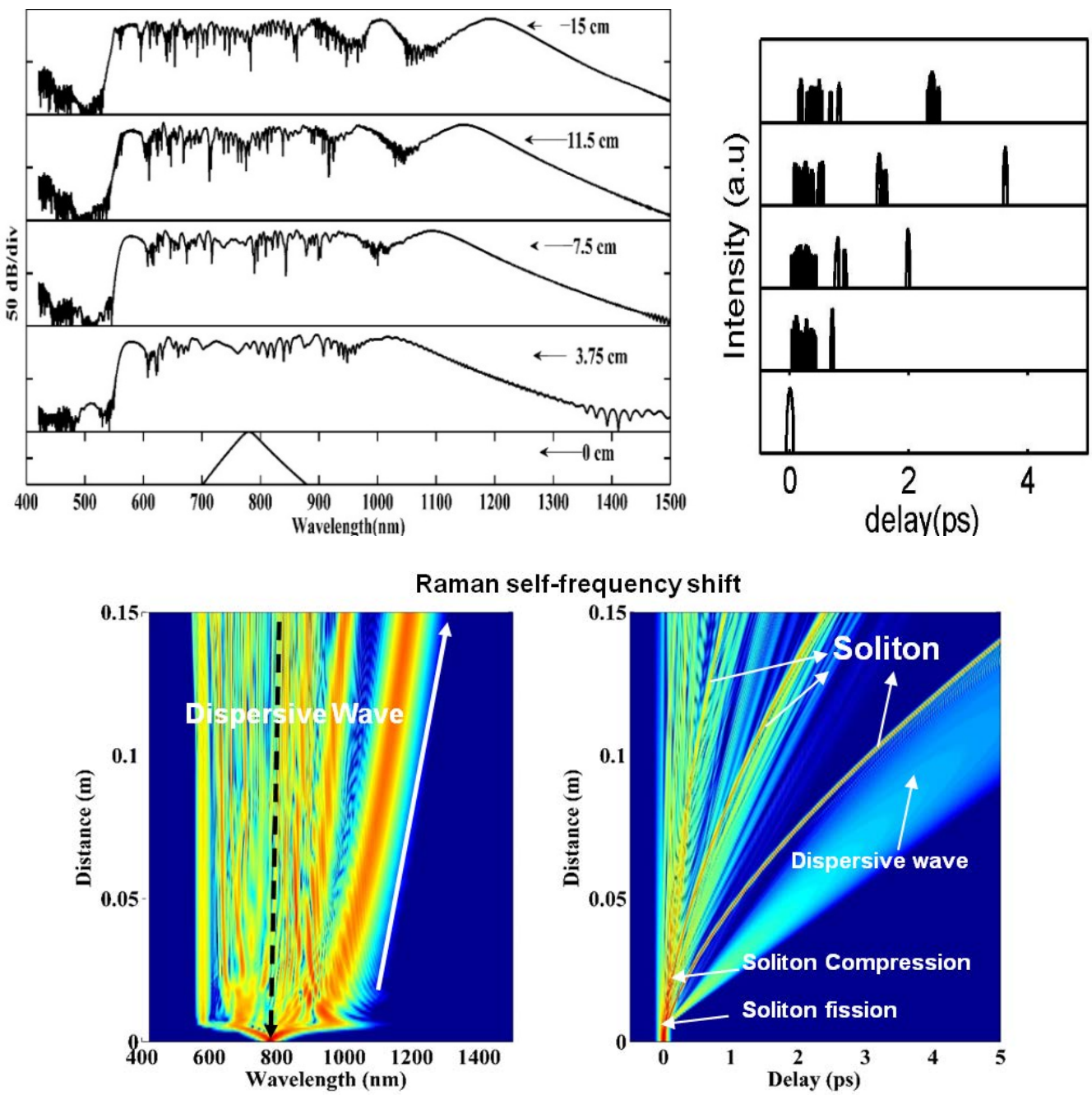

Figure 9. Result from the numerical analysis showcasing the SC generation in the proposed fiber (HNLF-2) with pump pulse centered around $780 \mathrm{~nm}$ (a) spectral evolution after $15 \mathrm{~cm}$ of the fiber (b) temporal evolution of the input optical pulse of $50 \mathrm{fs}$ FWHM. The subplots show the discrete slices at various lengths of the fiber.

Figure 9 illustrates the SCG during the propagation in a typical photonic crystal fiber. This soliton fission process explains the octave-spanning SCG in fibers. It is noteworthy to show in Figure 9, that the pulse starts its propagation as a high-order soliton at negative dispersion close to the ZDW. Because of the high TOD, the input soliton fissions into $\mathrm{N}$ fundamental solitons, which are subsequently Raman shifted toward the red. Each fission process gives rise to the emission of non-solitonic radiation at blue frequencies and the non-solitonic radiation propagates as a dispersive wave (Figure 10). As a result of these processes, the supercontinuum is created.

In SC generation, the presence of higher-order dispersion $\left(\beta_{3}\right)$ reshapes the soliton fission in two ways. The fundamental soliton ejected from the process generally shifts toward the shorter frequency with the contribution from the Raman shift (Figure 11). Second, the higher-order dispersion influences the transfer of energy from the fundamental toward the low amplitude temporal pedestal through linear and nonlinear soliton phase matching with the dispersive wave. Moreover, the initial emission of dispersive radiation is primarily from the first ejected soliton that possesses the broadest bandwidth and thus has maximum overlap with the dispersive wave resonance. Figure 11 demonstrates the resonant energy transfer with and without Raman contribution. The evolution of the dispersive wave due to the higher dispersion effectively modifies the propagation of the Raman soliton and further reduces the amplitude of the SSFS. 

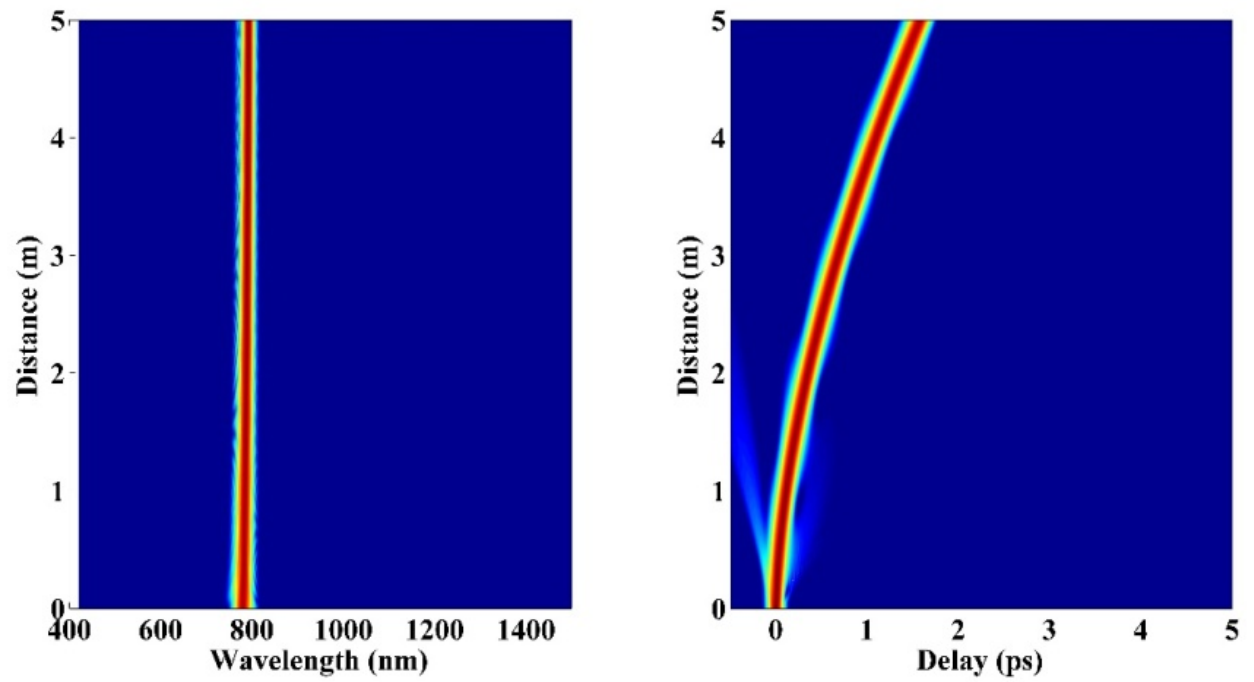

Figure 10a. Spectral and temporal evolution in the PQF for a length of $5 \mathrm{~m}$, with $\mathrm{N}=1 \mathrm{P}_{0}=66.252 \mathrm{~W}, \mathrm{~L}_{\mathrm{D}}=7.701 \mathrm{~cm}$, FWHM $=50 \mathrm{fs}$, Soliton length $\mathrm{z}_{\mathrm{sol}}=12.1 \mathrm{~cm}$.

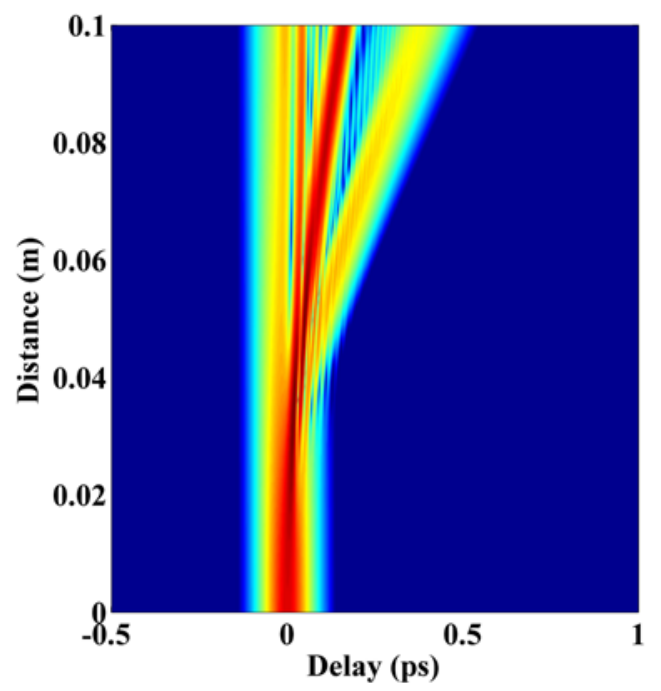

Figure 10b. Spectral evolution showing the soliton fission $\mathrm{L}_{\text {fiss }}=2.56 \mathrm{~cm}$ with $\mathrm{N}=3 \mathrm{P}_{0}=596.26 \mathrm{~W}, \mathrm{~L}_{\mathrm{D}}=7.701 \mathrm{~cm}$, FWHM $=50 \mathrm{fs}$, soliton length $\mathrm{z}_{\mathrm{sol}}$ $=12.1 \mathrm{~cm}$. 

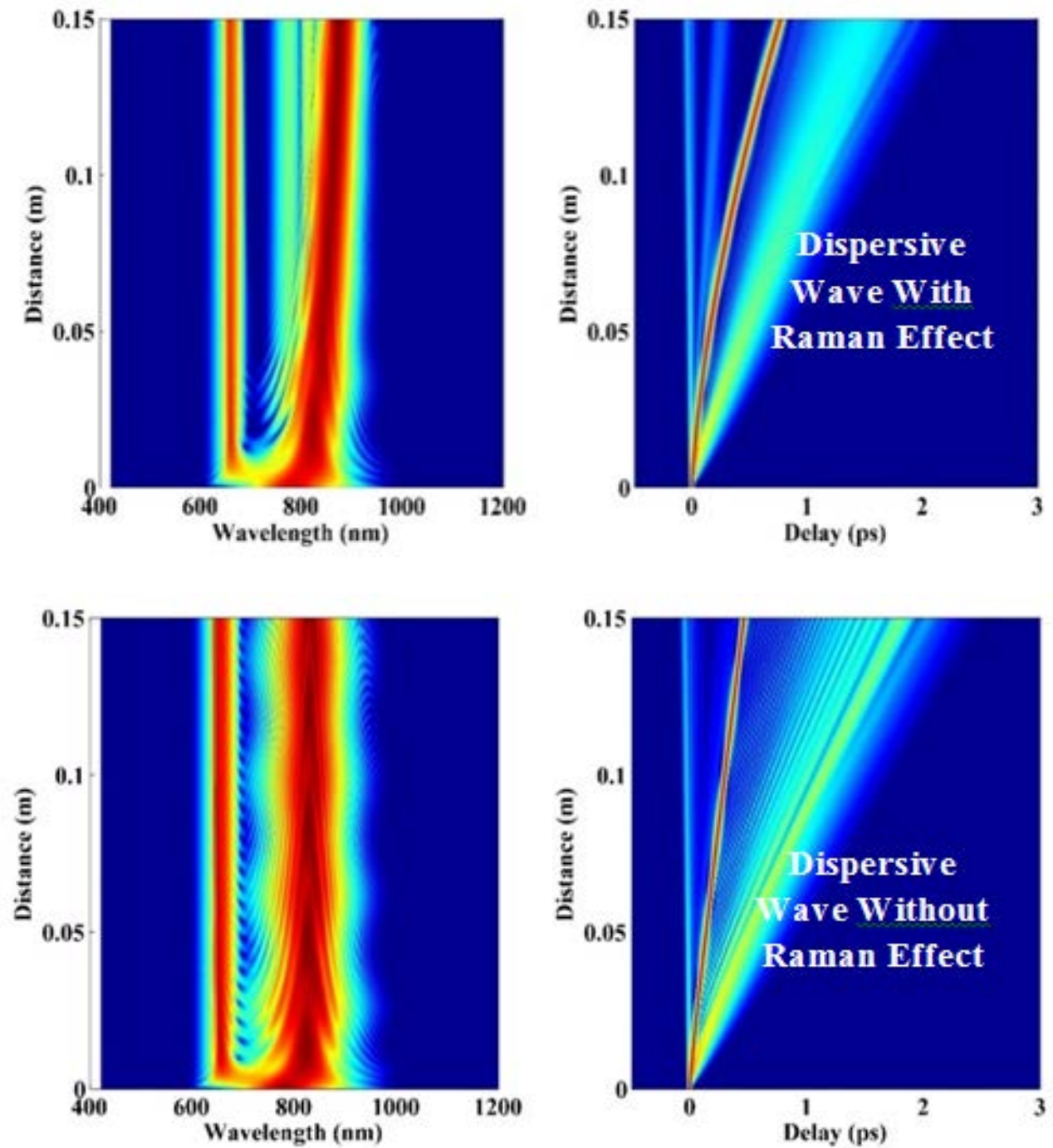

Figure 11. Spectral and Temporal evolution representing the impact of Raman scattering on dispersive wave dynamics at pump wavelength $780 \mathrm{~nm}$ with a peak power of $4.2 \mathrm{~kW}$ and FWHM of $10 \mathrm{fs}$.

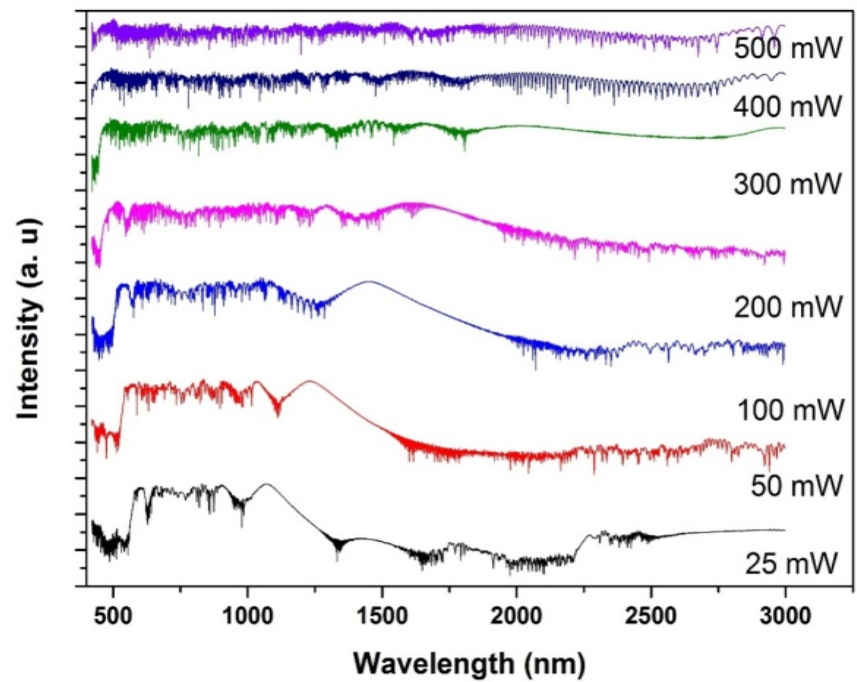

Figure 12. Supercontinuum spectrum observed in HNLF-2 with pump average power increasing from $25 \mathrm{~mW}$ to $500 \mathrm{~mW}$ at a wavelength of $780 \mathrm{~nm}$ with FWHM 50 fs. 

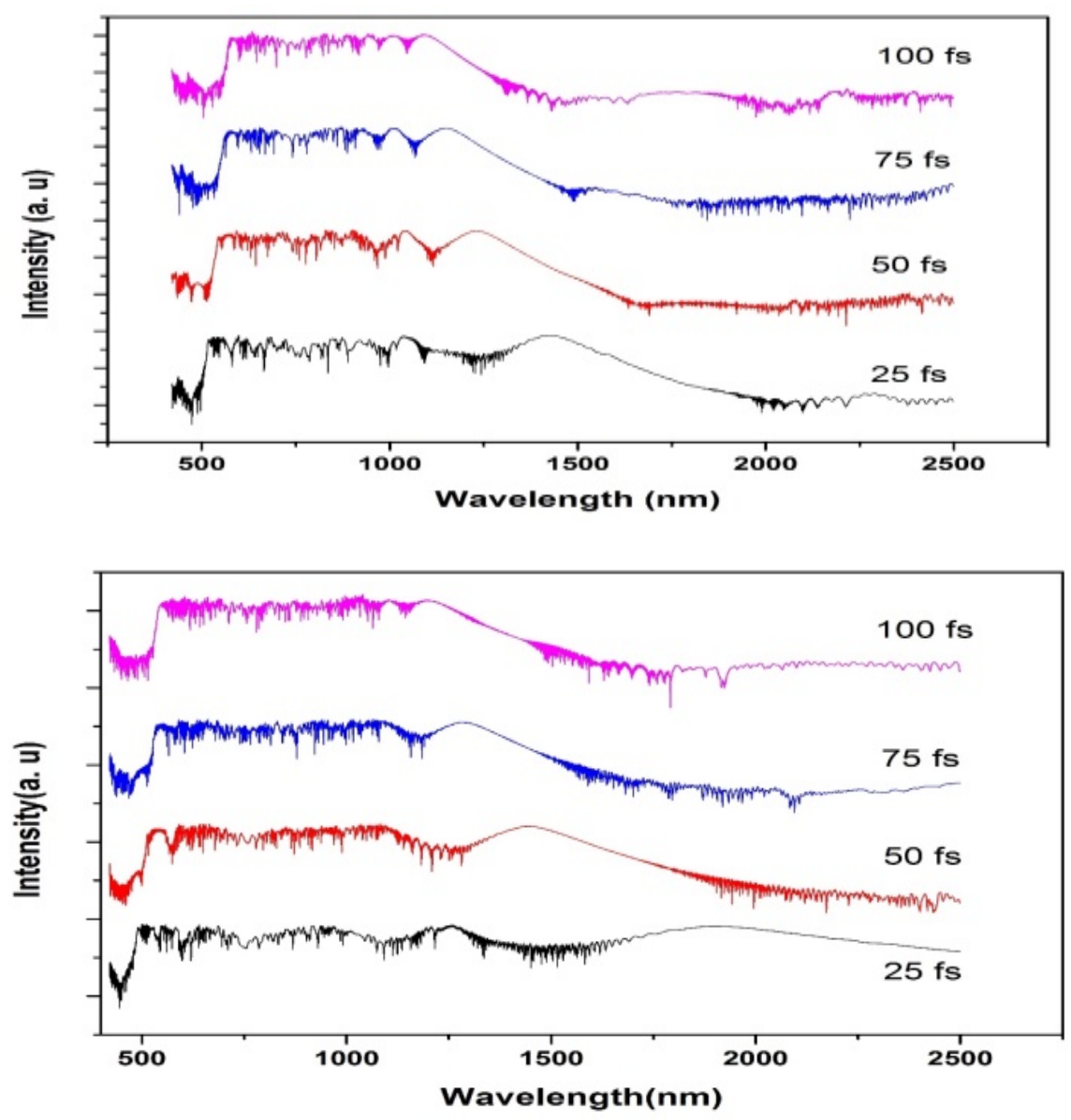

Figure 13. Impact of input pulse width on the spectral dynamics (a) average power $50 \mathrm{~mW}$ and pulse width FWHM 25 fs to 100 fs (b) average power $100 \mathrm{~mW}$ and pulse width FWHM 25 fs to $100 \mathrm{fs}$

The SC evolution with increasing pump peak power is illustrated in Figure 12. The pulse width for this analysis is kept constant at $50 \mathrm{fs}$ (FWHM) and the repetition rate of 80 $\mathrm{MHz}$, while the pulse energy is increased from $0.31 \mathrm{~nJ}$ to $6.25 \mathrm{~nJ}$. The useful spectral bandwidth $(-20 \mathrm{~dB}$ level) measured from the generated SC for the average power 50 $\mathrm{mW}\left(\mathrm{P}_{0}\right.$ the peak power is $\left.5.8 \mathrm{~kW}\right)$ is $536.67 \mathrm{~nm}$. When the average power reaches $200 \mathrm{~mW}$, the $-20 \mathrm{~dB}$ spectrum falls between $484.79 \mathrm{~nm}$ and $1747.49 \mathrm{~nm}$ with a bandwidth of $1262.7 \mathrm{~nm}$. When the peak power was increased to $117 \mathrm{~kW}$ (500 $\mathrm{mW}$ average power), the spectrum band width increased to $2726.4 \mathrm{~nm}$.

In Figure 13, the influence of pump input pulse duration on the evolution of spectral width is discussed. The average power for the pump pulse was chosen to be $50 \mathrm{~mW}$ and 100 $\mathrm{mW}$ increasing the pulse width duration from 25 fs to 100 fs (FWHM). Here, the analysis restricted to the anomalous pumping $(780 \mathrm{~nm})$ where the evolution of the SC is more significant. In both the cases, as the pulse duration increases the soliton order, the pulse energy increases directly in proportion to the average power from $0.32 \mathrm{~nJ}$ to $1.25 \mathrm{~nJ}$. The peak power used for these cases is $24.4 \mathrm{~kW}$, $11.7 \mathrm{~kW}, 7.8 \mathrm{~kW}$, and $5.87 \mathrm{~kW}$ to maintain the pulse energy constant at $0.32 \mathrm{~nJ}$ and $1.25 \mathrm{~nJ}$. The SC spectra intensities shown on the figures are plotted against the logarithmic scale and the useful bandwidth is measured for $-20 \mathrm{~dB}$ level. The bandwidth for the 25 fs pulse measures around $1002.6 \mathrm{~nm}$ and for the $100 \mathrm{fs}$ pulse $551.82 \mathrm{~nm}$. Similarly for the second case with average power $100 \mathrm{~mW}$, the useful bandwidth for $25 \mathrm{fs}$ and $100 \mathrm{fs}$ is $1633.59 \mathrm{~nm}$ and $685.53 \mathrm{~nm}$, respectively. 


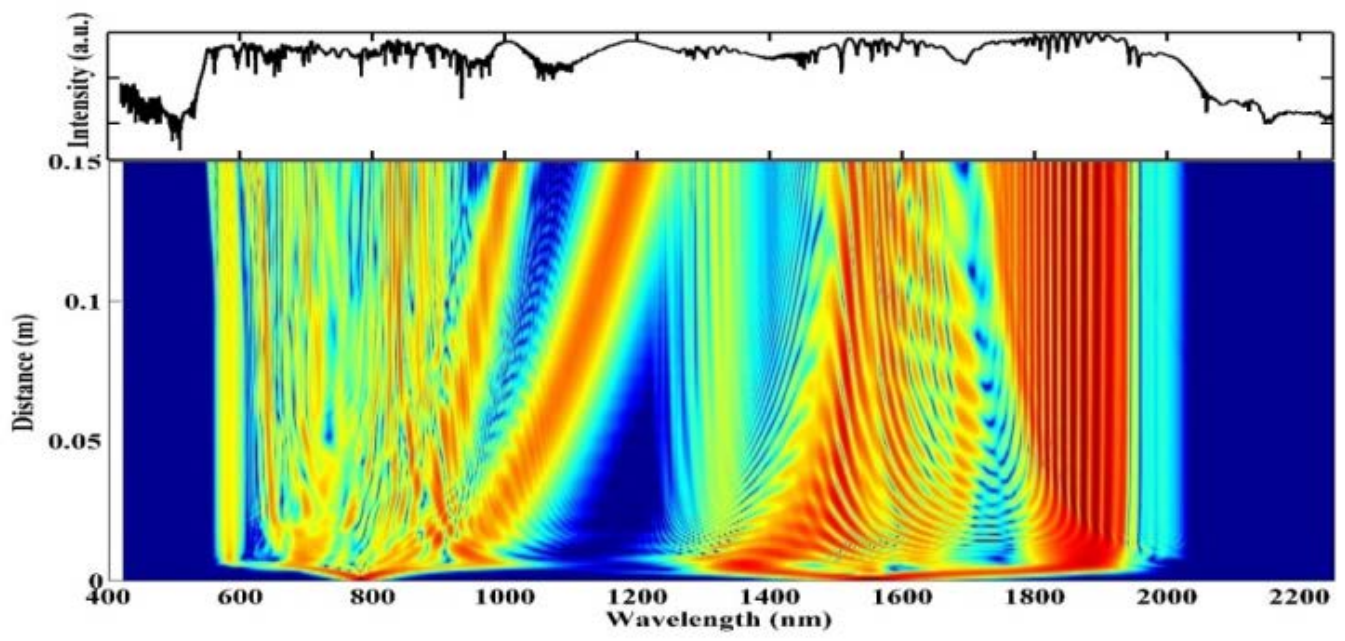

Figure 14. SC generation by dual wavelength pump in PQF with two widely located ZDWs

In 2009, Xiong et. al., has demonstrated a dual pump configuration at the wavelength of $686 \mathrm{~nm}$ and $1064 \mathrm{~nm}$ generating a spectrum extending from $370 \mathrm{~nm}$ to 1750 $\mathrm{nm}[21]$. Similarly, the generation of SC in dual ZDW [10, 22], both located in anomalous region generating a bandwidth extending from $426 \mathrm{~nm}$ to $2954 \mathrm{~nm}$ is been investigated. Encouraged by this report, two pump pulses of wavelength at $780 \mathrm{~nm}$ and $1550 \mathrm{~nm}$ are launched into the fiber. Both the pump pulse falls within the anomalous dispersion regime. The pulse duration is kept constant at 50 fs (FWHM) and the peak power at $10 \mathrm{~kW}$ enhancing the interaction between the two co-propagating pulses and thus extending the spectral bandwidth from $536 \mathrm{~nm}$ to $1395 \mathrm{~nm}$ for a propagating distance of $15 \mathrm{~cm}$ (Figure 14). When the pump power was increased to $100 \mathrm{~kW}$, the spectrum extended from $459 \mathrm{~nm}$ to $3040 \mathrm{~nm}$.

\section{Conclusions}

In summary, a PQF with nonlinear coefficient of 196 $\mathrm{W}^{-1} \cdot \mathrm{km}^{-1}$ at $780 \mathrm{~nm}$ and a broad anomalous dispersion regime with a bandwidth of $908 \mathrm{~nm}$ are designed for SC generation. The influences of the pump pulse parameters, pump pulse width and average power on the bandwidth of the generated SC are studied. This study has determined the optimum pump conditions for the SC generation in these proposed fibers. In this simulation, the pulse width of $50 \mathrm{fs}$ with a $\underline{\mathrm{P}}_{\text {avg }}$ of $500 \mathrm{~mW}$ and with pulse energy $6.25 \mathrm{~nJ}$ is considered for pulse propagation through a $15 \mathrm{~cm}$ long PQF generating a maximum SC bandwidth of $\sim 2726 \mathrm{~nm}$. Moreover, this analysis exhibited various geometrical parameters influencing the dispersion profile and thereby variation in the generation of SC. To conclude, many applications such as optical coherence tomography, frequency combs, and ultrashort pulse generation will find a tremendous advantage with these highly coherent, large broadband light sources.

\section{REFERENCES}

[1] Alfa R R and S L Shapiro 1970 Phys. Rev. Lett.245 84-587

[2] Ien EP and Stolen R H 1972 Appl. Phys. Lett.21539-541

[3] Stolen R H and Ien E P 1973 Appl. Phys. Lett.22 6 276-278

[4] Stolen R H and Lin C 1978 Phys. Rev. A17 4 1448-1453

[5] Saitoh K, Koshiba M, Hasegawa T, and Sasaoka E 2003 Opt. Express11 8 843-852

[6] Washio K, Inoue K, and Tanigawa T 1980 Elect. Lett. 16 331-333

[7] Bétourné A, Kudlinski A, Bouwmans G, Vanvincq O, Mussot A, and Quiquempois Y 2009 Opt. Lett 34 3083-3085

[8] Zhang R, JörnTeipel and Harald Giessen 2006 Opt. Express14 6800-6812

[9] Federico Belli, Amir Abdolvand, Wonkeun Chang, John C Travers, and Philip St J Russell 2015 Optica 2 292-300

[10] Tongtong Zhao, Shuqin Lou, Wei Su, and Xin Wang 2015 J. Mod. Opt.63139-145

[11] Birks T A, Knight J C and Philip St J Russell 1997 Opt. Lett.22 13 961-963

[12] Andersen T V, Hilligs $\varphi$ e K M, Nielsen C K, Thøgersen J, Hansen K P, Keiding S R and Larsen J J 2004 Opt. Express12 4113-4122

[13] Levine D and Steinhardt P J 1984 Phys. Rev. Lett.53 26 2477-2480

[14] Sircilli F MAR Franco and Serrão VA 2007 EUROCON The International Conference on Computer as a Tool, Warsaw Poland 1263-1268

[15] Bahrampour A, Iadicicco A, Foomezhi E, Momeni S, and Bhrampour AR 2014 Photonics Conference Third 
Mediterranean 1-3

[16] Aliramezani M and Mohammad NejadS 2010Opt. Laser Techl.42 1209-1217

[17] Hilligsøe K M et al., 2004 Opt. Express12 1045-1054

[18] Dudley J M, and Taylor J R 2010 Cambridge University Press

[19] Ranka J K, Windeler R S, and Stentz A J 2000 Opt. Lett. 25 25-27

[20] Wang Y, Hou J Y, Xiong C, Peng Y, Song R B, Eggleton B J, and Lu Q 2010 IEEE Asia Communications and Photonics

[21] Xiong C, Chen Z, and William J Wadsworth 2009 J. Light. Technol.27 1638-1643

[22] Peng Chen et al., 2018 Appl. Phy. B 123128:1-7

[23] Sivacoumar R, and Alex Z C 2015 Optoelectronics and Advanced Materials - Rapid Communications9 1208-1213 